\title{
Trade-offs in Time-to-Digital Converter Architectures for Harsh Radiation Environments
}

\author{
Bjorn Van Bockel, Paul Leroux, and Jeffrey Prinzie
}

\begin{abstract}
Performance degradation of standard time-to-digital converters is inevitable due to the effects of ionising radiation. In this article, the trade-offs of different mitigation techniques for time-to-digital converter architectures are presented. As the effect of radiation is challenging for such high-performance circuits in the state of the art technologies, architectures that can compensate for radiation effects have become a necessity for TDCs to operate in these harsh radiation environments. By looking into the functionality of different architectures focusing on improving radiation tolerance, the main weak and strong points of different time-to-digital converter architectures, including the compensation circuitry, are described. Possible solutions to those weak points are presented.
\end{abstract}

Index Terms-Delay locked loop (DLL), enclosed layout transistor (ELT), gated ring oscillator (GRO), multi-stage noise shaping (MASH), phase locked loop (PLL), radiation hardened, time-to-digital converter (TDC), total ionising dose (TID), triple modular redundancy (TMR)

\section{INTRODUCTION}

$\mathbf{T}$ IME-TO-DIGITAL converters (TDC) are electronic circuits used to convert timing events (time differences) into a digital representation. A basic representation of a Flash-type TDC is shown in Figure 1. Already in the 1960s, TDCs were introduced in the design of nuclear reactor technology and instrumentation. More specifically, TDCs were used to measure the neutron velocity (hence its energy) to determine the energy spectrum of different materials, using a Time-of-Flight (TOF) neutron detection. Since semiconductor technologies, at that time, were not as efficient as today's technologies, time measurements were limited to microseconds resolution and were mainly implemented by counting the oscillators clock cycles [1]. Later, TOF measurements were used for particle tracking, and detection, accurate positioning, distance measurements and coincidence counting [2].

As Complementary Metal Oxide Semiconductor (CMOS) technologies have scaled-down, the supply voltage headroom has decreased significantly down below $1 \mathrm{~V}$, which makes it increasingly difficult to design voltage based analogue-todigital converters (ADCs) [3]. Therefore operating entirely in the time domain, by use of direct time measurement, has become more interesting [4], [5].

The following three parameters mainly determine the performance of a TDC: (1) Conversion step size, also reported as bin size or resolution, which describes the ideal time discretization step of the TDC. (2) Power consumption, which for some

B. Van Bockel, P. Leroux and J. Prinzie are with with the Department of Electrical Engineering, KU Leuven, Belgium. e-mail: bjorn.vanbockel@kuleuven.be

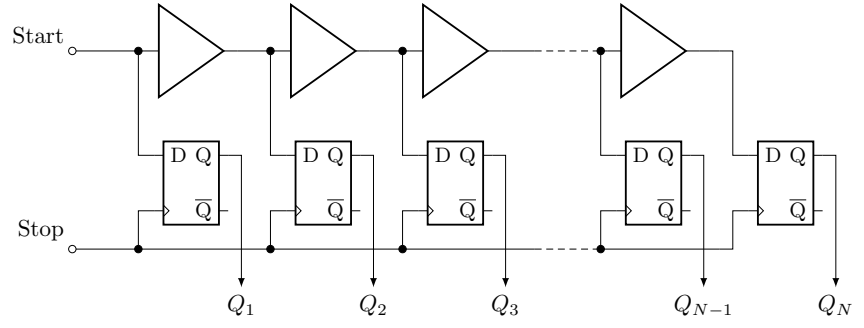

Fig. 1. Flash TDC, Basic architecture of a time to digital converter. A signal coming from a start event propagates the delay line, which is sampled by a signal coming from a stop event

architectures is related to the duration of the input signal. (3) The measurement range (dynamic range), describing the maximum time interval a TDC can measure. It also determines the maximum sample frequency of the TDC. Depending on the application, a trade-off between these parameters needs to be made to suit the application.

One particular example where TDCs are used for particle detection application is Positron Emission Tomography (PET). Radioactive tracers, emitting positrons, inside cancer patients can reveal the location of the cancerous tissue. These positrons annihilate in the tissue emitting two identical gamma rays with opposite momentum. These gamma rays are detected by a circular detector around the patient, consisting of hundreds of individual channels. Since the direction of the emitted photons is random, images can be formed by statistically combining many samples, backtracking the emission point, which was how conventional PET worked without considering any timeof-arrival information [6]. However, the imaging resolution can be significantly improved by considering the arrival time of the photons. Henceforth, the difference of TOF between the detected gamma rays relates to the position and distribution of cancer tissue inside the patient's body [7].

Another application of TOF can be found in navigation, positioning systems and many autonomous vehicles and robots. They are equipped with a 2D or 3D LIDAR (LIght Detection And Ranging) that continuously scans the room by emitting a laser beam, which corresponds to the start trigger, and receives the reflected beam, corresponding to the stop trigger. The time difference between those two signals relates to the distance between the vehicle and the object (TOF) at which the laser beam was reflected. Complex algorithms such as Simultaneous Localization and Mapping (SLAM) can autonomously sense the environment and update the most probable position, which is used as the main instrument for indoor navigation [8].

As part of a larger circuit, a more integrated application 
is the All-Digital Phase-Locked Loop (ADPLL). Driven by technology scaling, Phase-Locked Loop (PLL) architectures shift towards fully digital techniques, replacing the phase detector, analogue charge-pump and loop-filter with digital circuitry, reducing area, variability and noise. In an ADPLL, the phase measurement is performed by a TDC, and the digital loop filter uses the digital output value to correct the oscillator [9].

Finally, High Energy Physics (HEP) detectors strongly rely on TDCs, where the detection of a particle related to a reference time is essential. This is the case in the Large Hadron Collider (LHC), where the time between the bunch crossing and the detection of a particle inside a detector needs to be measured very precisely, especially for the planned HLLHC where an increased collision rate makes it increasingly difficult to reconstruct the physics events [10], [11]. Additionally, TDCs are also used in Time over Threshold (TOT) measurements. Where they are used for measuring drift times inside drift tube detectors and Gas Electron Multiplier (GEM) detectors (Figure 2), to quantify the energy of the detected particles.

The applications mentioned above operate in harsh environments which can be defined by different environmental conditions presented in an extreme way. This can be operating temperatures beyond $-40^{\circ} \mathrm{C}$ to $125^{\circ} \mathrm{C}$, exposure to hostile chemical environments like saltwater or high humidity. However, this paper will focus on the environmental element of ionising radiation, which occurs in nuclear energy applications like nuclear fission or fusion and high energy physics experiments and applications in space and deep space. Hence, TDCs are required which can operate under these demanding conditions. The different factors which can have a significant impact on the performance of TDCs are temperature variations, supply voltage variations and ionising radiation. Different techniques

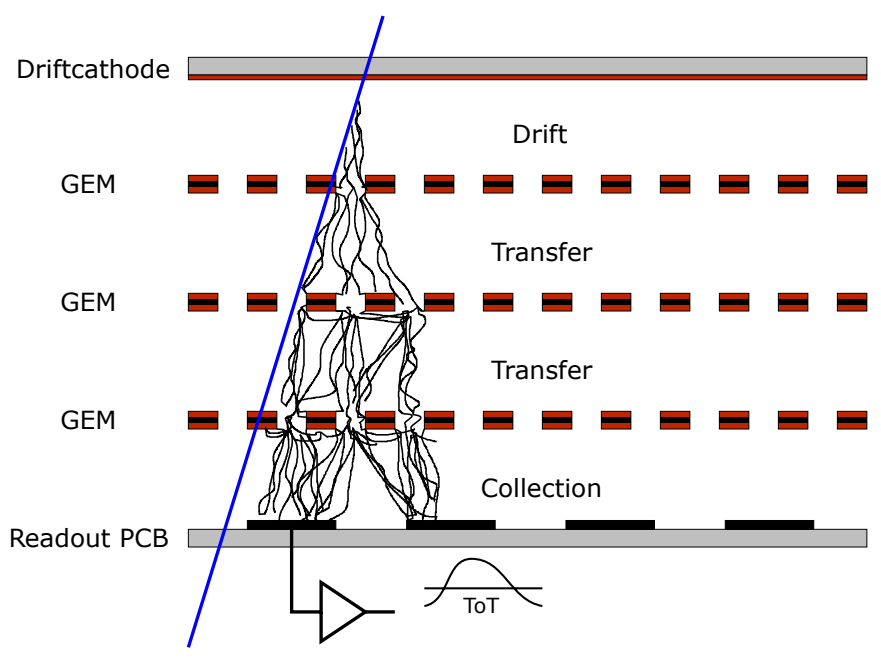

Fig. 2. Cross-section of a triple GEM detector. Ionisation electrons are produced by a charged particle crossing the GEM detector in the drift gap. Due to a large electric field across the GEM foils, the electrons are attracted towards the readout PCB. When electrons pass one of the GEM foils, the local electric field, which is $\sim 100$ times the field present in the drift region, causes a multiplication of the electrons, which can be more easily detected by the readout electronics. [12].

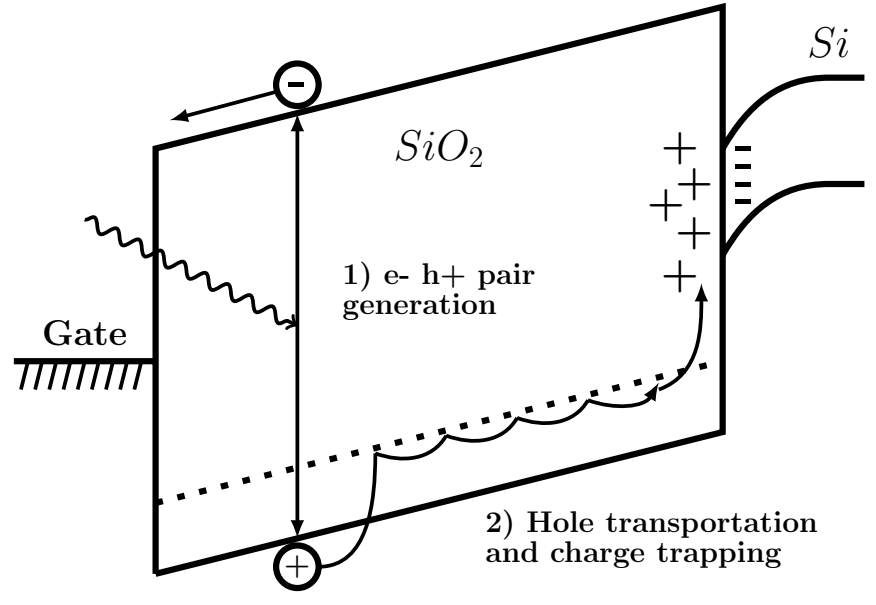

Fig. 3. Energy band diagram of a MOS structure with positive gate bias and the mechanism of positive charge migration and trapping due to ionising radiation [13].

exist to improve reliability under these circumstances. The scope of this paper is to describe different compensation techniques for the effects of ionising radiation through several techniques that simultaneously correct temperature and supply voltage variations.

\section{EFFECTS OF IONISING RADIATION ON TDCS}

The effects of ionising radiation on CMOS devices can be split into two main categories. The long-term cumulative effects, Total Ionising Dose (TID) effects, which cause degeneration and in some cases complete failure of devices, and the short-term effects, called Single-Event Effects (SEEs), which can cause instantaneous disturbances (soft-errors) or even failures due to destructive events.

TID effects are cumulative effects that will gradually degrade the properties of CMOS devices due to trapped charges in the oxide insulation layers of the transistor and semiconductor to oxide interfaces. When CMOS devices are exposed to ionising radiation, trapped charges in the $\mathrm{SiO}_{2}$ and at the silicon to $\mathrm{SiO}_{2}$ interfaces, as shown in Figure 3, impact the electrical field in the device. Conventionally, trapped charges in the gate oxide cause the threshold voltage to shift, which was one of the most pertinent TID effects in older CMOS technologies [14]. In new deep submicron devices, the oxide thickness has shrunk such that most degradation results from trapped charges in secondary insulators such as the SiN spacers, Shallow Trench Isolation (STI) or Buried Oxides (BOX) in Silicon-on-Insulator (SOI) devices [15]-[17]. For TDCs, this effect reduces the speed of the transistor, generally causing the converter step size to increase, resulting in a reduced resolution. As TID effects also amplify local mismatch, as described in [18], the non-linearity errors will also increase, degrading the Effective Number of Bits (ENOB) of the converter.

Contrary to TID effects, which are cumulative, SEEs are instantaneous [19]. They result from the interaction of a high-energy particle with the silicon, which generates free carriers in the silicon, which in turn can be captured near 


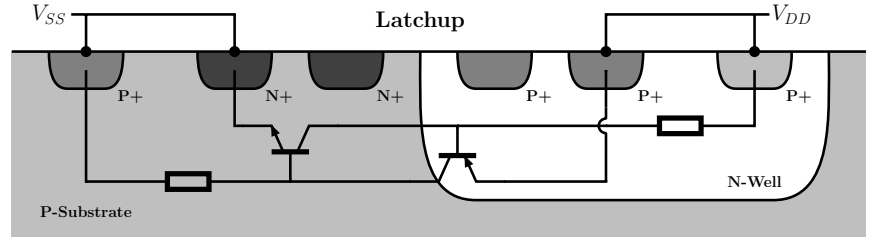

Fig. 4. Cross section and equivalent circuit of the parasitic pnp and npn transistors in CMOS circuits forming an SCR.

the MOS junctions, causing large currents to flow through the junctions for several hundreds of picoseconds. This current can temporarily disturb signals on the chip and can flip the logic state of a digital storage element or trigger the Silicon Controlled Rectifier (SCR) pnpn-structure, shown in Figure 4 , which shorts the power supply and can damage the chip [20]. Furthermore, Single Event Upsets (SEUs) can cause the output to be unreliable and Single Event Transients (SETs) can temporarily disturb a compensation loop locked state.

In order to design a suitable TDC architecture for radiation environments, it is important to understand these mechanisms and use Radiation Hardened By Design (RHBD) techniques to mitigate these effects [21], [22], as will be described in the next section.

\section{RADIATION HARDENING BY DESIGN TECHNIQUES}

Generally, several types of RHDB techniques are possible. Either structural (circuit independent) or ad-hoc. RHBD techniques which do not depend on the architecture, are mostly layout specific techniques. As described in [23], [24], using the Enclosed Layout Transistor (ELT) or ' $\mathrm{H}$ ' layout reduces the degradation effects of ionising radiation. As these transistors have a larger minimum size than regular transistor layouts, area and power consumption will generally be larger due to design rule restrictions.

A commonly used technique to mitigate SEEs in digital circuits is Triple Modular Redundancy (TMR). TMR is implemented by triplicating all logic cells. If one cell or module is corrupted due to an SET or SEU, voters at the output will select the correct output by majority voting. As shown in Figure 5, the voters are also triplicated because they are also sensitive to SEUs. A full TMR implementation consists of three inputs, three outputs, three identical digital modules and three voters. Because every logic cell is triplicated and extra logic is added, it can be seen that both area and power will at least increase by a factor of three [22], [25]. The final output voters will always remain a sensitive circuit and can by definition not be triplicated. These circuits need to be hardened through dedicated logic implementation [26], transistor sizing, the use of dummy devices for charge sharing [27], filtering and intelligent exploitation of the pulse quenching effect [28].

\section{Degradation mitigation techniQues}

In the design of RHBD TDCs, it is often difficult or even impossible to monitor the bin size and adjust the performance of the TDC by performing an intermediate offline calibration by inspection. Therefore, automatic offline or online calibration and compensation are required. We will elaborate on two main techniques (a) Code Density Test (CDT) with Lookup Table (LUT) adjustments and (b) feedback loop compensation to guarantee the bin size of the TDC.

\section{A. Code Density Test}

A CDT is commonly used to statistically determine the bin size of a TDC since it is difficult to precisely perform a sweep of an input time delay that covers the complete dynamic range. With a CDT, an uncorrelated delay generator source generates a set of random input samples covering the full dynamic range of the TDC. This can be easily implemented with a noisy external clock generator to achieve a uniform input distribution. Since the input delays are random and uncorrelated to any reference signal in the TDC, the hit probability of each bin is only dependent on the specific bin size. The output of this test will thus generate a Probability Density Function (PDF) which corresponds to the bin size of each bin. This can be accumulated to a Cumulative Density Function (CDF), forming the transfer function of the TDC. This transfer function can then be used to build a LUT to compensate for possible nonlinear imperfections in a postprocessing stage [29].

A critical consideration of the CDT method is that this technique allows calibrating or compensating imperfections between conversion steps relatively. However, it does not provide any information on the absolute conversion step size or TDC gain, which will also degrade due to ionising radiation. To measure the conversion step size, a fixed time-interval input signal must be applied and measured and based on the average output, the absolute conversion step size (i.e. TDC gain) can be calculated. Since it is beneficial to have a stable conversion step size during the lifetime of the TDC, which cannot always be guaranteed in harsh environments, continuous monitoring should be done. Alternatively, an active feedback method can be used.

\section{B. Feedback}

Feedback techniques are commonly used for online calibration. In most cases, this technique uses a loop structure to control the timing generator blocks in a TDC to be stable over time. This was done in [30], [31] where the delay line or ring oscillator is placed inside a Delay Locked Loop (DLL) or Phase Locked Loop (PLL), respectively. By using this technique radiation degradation of $15 \%$ could be compensated

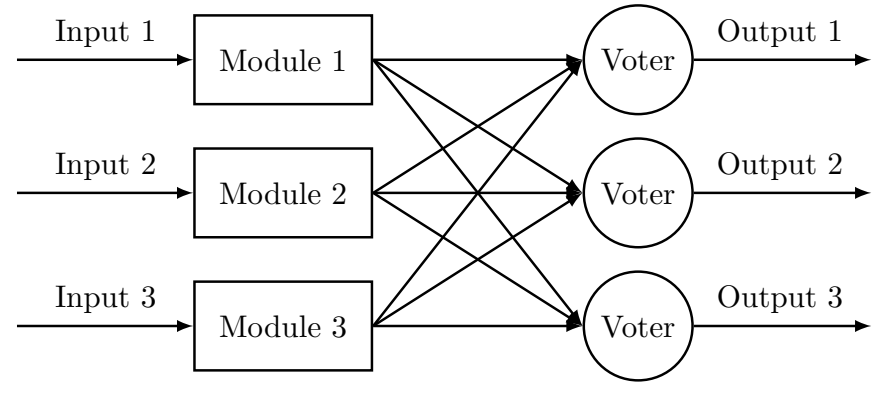

Fig. 5. Tripple modular redundancy technique. 
up to a dose of 2.2MGy. Apart from a locked loop structure, a replica delay line can also compensate the delay cells in a flash TDC architecture. This allows propagating the start signal and sample on the stop signal, reducing the quantization error compared to a locked loop architecture for absolute measurements.

The advantage of online feedback is continuous compensation without requiring a special calibration sequence, which makes the TDC available for measurements at any time. Since the feedback loop must compensate for degradation (delay increase) in the future, the achievable initial performance of this type of TDC will always be less than the maximal TDC performance without any compensation since additional tuning margin is needed to counteract radiation degradation, envisaged ahead. As these loop structures can be different for every architecture, they need to be analyzed individually depending on the performance requirements.

As the effects of ionising radiation impact the entire chip, they also impact the associated calibration algorithm, feedback structure, and TMR implementation. Degradation of these circuits generally decreases the compensation dynamic range or introduces static offset and an increased random noise contribution. Therefore a clear trade-off between using a more bare-bones architecture or adding compensation circuits needs to be made. Concluding from the techniques mentioned above, it can also be seen that there is a clear trade-off between improved radiation tolerance and area and power consumption. In the following sections, different architectures are discussed where RHBD is applied.

\section{LOCKED LOOP ARCHITECTURES}

Relatively simple but very robust architectures are the locked loop architectures. These architectures, as shown in Figure 6, consist out of a chain of tunable delay cells either as a delay line or as a ring oscillator for a DLL or PLL, respectively. They operate as a reference timing generator for the TDC.

In a DLL based TDC, the delay line is locked to the period of a reference clock connected to the input of the delay line. The output is fed back to a phase detector to detect whether the delay line output leads or lags the reference clock. This error is then processed by the Charge Pump (CP), which will charge

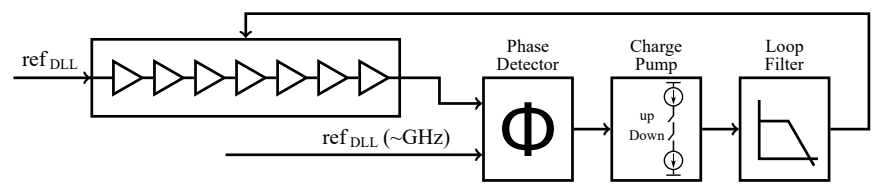

a) Delay Locked Loop

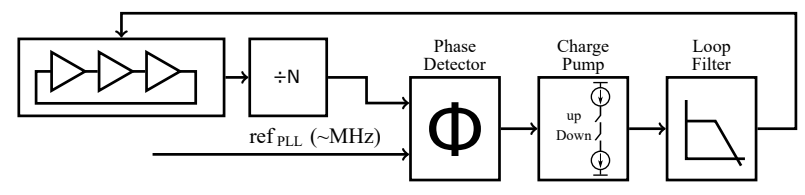

b) Phase Locked Loop

Fig. 6. (a) Delay Locked Loop (DLL) architecture. (b) Phase Locked Loop (PLL) architecture. or discharge a loop filter to adjust the control voltage of the tunable delay cells. When the loop is locked to the reference clock, the total delay of the delay line precisely matches the reference period. A drawback of this approach is the need for a relatively high-frequency reference clock to limit the length of the delay line. Long delay lines would result in large nonlinearities. Therefore, in practice, DLLs are often preceded by a radiation-hardened, stable, low phase noise PLL with inductor based VCO instead of a ring oscillator, to synthesise a reference signal [32].

The PLL based TDC is similar to the DLL TDC. The Voltage Controlled Delay Line (VCDL) is replaced by a Voltage Controlled Ring Oscillator (VCRO). A reference clock is still used to lock the phase and, therefore, also the frequency of the VCRO. In this architecture, the different phases of the ring oscillator are used to generate the timing information of the input triggers. In contrast to DLLs, the reference clock period does not need to be equal to the period of the VCRO, which is beneficial as the VCRO period can be relatively small and would otherwise require a very high-frequency reference clock, which is impractical (typically reference crystals are available up to $100 \mathrm{MHz}$ ). However, when a measurement across multiple VCRO periods is done, the phase noise of the ring oscillator will introduce accumulated jitter in the measurements, which is not the case for a DLL architecture. In a DLL architecture, the reference clock is not circulated inside the delay line but is synthesised by a low phase noise LC PLL, typically 10x better (lower jitter) or more than ring structures.

The Phase Detector (PD), Charge Pump (CP) and Loop Filter (LF) form the feedback loop, which keeps the conversion step size fixed. However, these circuits are also exposed to ionising radiation, causing them to degrade. For PDs, SEEs are more problematic than TID effects since an inaccurate phase detection can disturb the loop's lock state, resulting in unreliable measurements. Therefore, TMR is commonly used to avoid false phase detection. PD's are either implemented as a Phase Frequency Detector (PFD) or binary Bang-Bang Phase Detector (BBPD). A PFD can be represented as a state machine, where an SEE can cause the state machine to jump into an undesirable state from which it cannot recover. In [33] a custom PFD architecture is presented which can recover from this faulty state.

BBPDs provide a non-linear output that can be seen as saturation, and they are memoryless. Henceforth, SEUs in the BBPD are not as catastrophic as in PFDs, since the state recovers each cycle independent of the previous state. Similarly, CDR (clock-data-recovery) detectors use no TMR since errors only persist for one cycle and are refreshed. The drawback of BBPDs is the limited pull-in range, requiring a frequency detector for initial acquisition [34]. For TID mitigation, wide devices or ELT transistors are used to limit speed degradation due to accumulated doses [35]. Reduced drive strengths will increase PD jitter. Hence the in-band phase noise of the PLL/DLL and, in turn, the effective single-shot resolution of the TDC reduces.

There is a difference in design methodology for a charge pump between designing for Process-Voltage-Temperature 


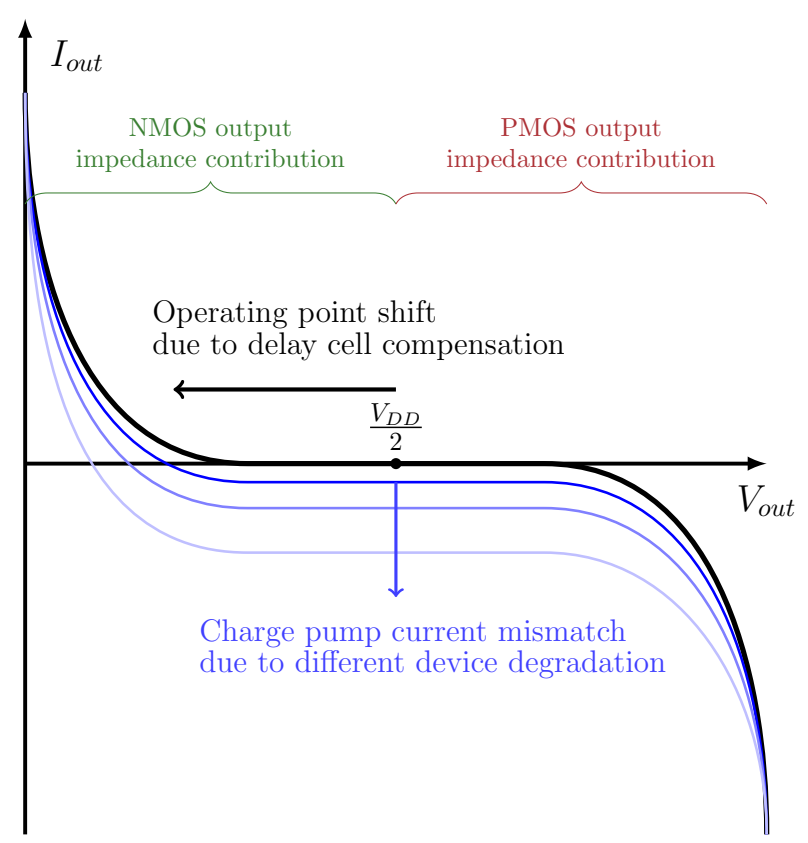

Fig. 7. Charge pump output characteristic. Shown in blue the current mismatch due to ionising radiation

(PVT) variations and effects of ionising radiation. PVTvariations are expected to be bound to the operating conditions and result in a relative variation around the set point. When the standard variations are known, they can be used to adjust the design accordingly.

For the effects of ionising radiation, this is different. As it is known that radiation degradation of the circuits is cumulative, the quantity of this variation depends on geometry, biasing conditions and the received dose. Moreover, the direction in which a circuit will degrade due to ionising radiation can be known from early simulation. Therefore, a different setpoint compared to the one used to compensate for PVT variations, which can be asymmetrical, may be chosen.

For the $\mathrm{CP}$, this means that the output control voltage will gradually shift toward one of the supply rails, away from the ideal operating point of the output stage, shown in Figure 7. As the output control voltage moves towards the threshold voltage of either the PMOS or NMOS current source, the difference between the two currents, due to the changing output impedance, will start to increase as the devices will enter the linear region. This results in the CP's inability to tune the output control voltage anymore and introduces a large Static Phase Offset (SPO). When using a PFD compared to a $\mathrm{BBPD}$, even a small current mismatch around the operating point of the $\mathrm{CP}$ will already introduce an SPO. For a PLL based TDC, this only changes the phase relation between the reference clock and the VCRO and will not change the frequency of the VCRO, therefore, not affecting the bin size. However, For a DLL based TDC, the SPO will result in a difference between the reference period and the total delay of the delay line, causing the bin size of the TDC to be changed.

The effects of ionising radiation increase the charge-pump

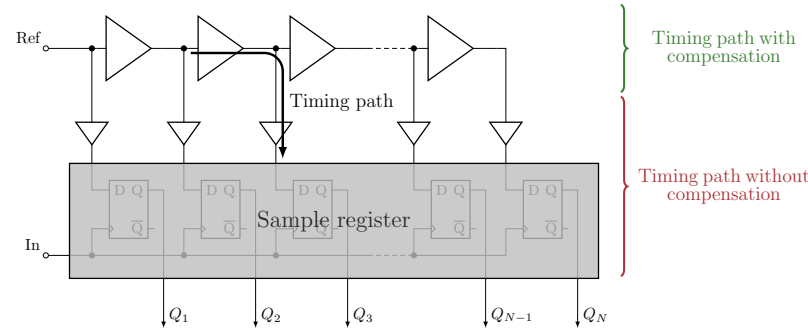

Fig. 8. Representation of the critical timing path of a delay element based TDC.

mismatch because different types of transistors degrade differently [35]. As these effects are cumulative for increasing dose, the CP will only work up to a specific limit at which it cannot compensate the delay cells accordingly, posing a TID dose limit. To improve the dynamic range of the $\mathrm{CP}$, a technique presented in [36] and [37] uses a replica circuit to tune one of the current sources to be equal to the other current source for the required set point, eliminating the possible current mismatch caused by PVT variations or ionising radiation. Another solution is to improve the compensation dynamic range requirements of the feedback loop. For example, to use digital calibration to coarsely set the delay and only rely on the $\mathrm{CP}$ for fine-tuning. In this way, the $\mathrm{CP}$ is always within bounds set by the coarse tuning resolution.

The loop filter used to store the charge of the $\mathrm{CP}$, resulting in a control voltage for the delay elements, generally consists of passive components. Therefore, the effects of ionising radiation are minimal and can be neglected. Note, however, that MOS capacitors (MOSCAPs) intrinsically leak, resulting in static phase offsets. This leakage can dramatically increase with TID. Henceforth, only metal capacitors or thick gate MOSCAPs are used in practice. Alternatively, fully digital filters are gaining more momentum in the field due to their intrinsic resistance against TID effects and other environmental variations.

While the feedback loop constantly tunes the delay line or oscillator to a fixed delay or frequency, the average delay cell mismatch is also stabilised in a first order. However, the clock distribution from the DLL/PLL timing generator to the sampling registers, shown in Figure 8, introduces another path for degradation. As the control loop does not compensate for the delay for this path, the overall nonlinearity of the TDC will increase with dose. [31].

Assessing the main performance properties for this architecture, we can observe that the resolution is mainly determined by the delay line, which is dictated by the individual delay elements. Smaller propagation delays of the delay elements will eventually result in an improved resolution of the TDC. The simplest delay element is a single inverter stage where the propagation delay is limited by technology. However, because of the inverting property, further readout becomes more complex, which lead to the use of differential or pseudo-differential delay cells [38]. This keeps the non-inverting property without increasing the conversion step size and improves the Power Supply Rejection Ration (PSRR), a well-known property of 
differential circuits. Moreover, using a proper differential delay cell, like the Maneatis cell [39], will result in an improved bin size due to the lower signal swing, although at the cost of higher jitter.

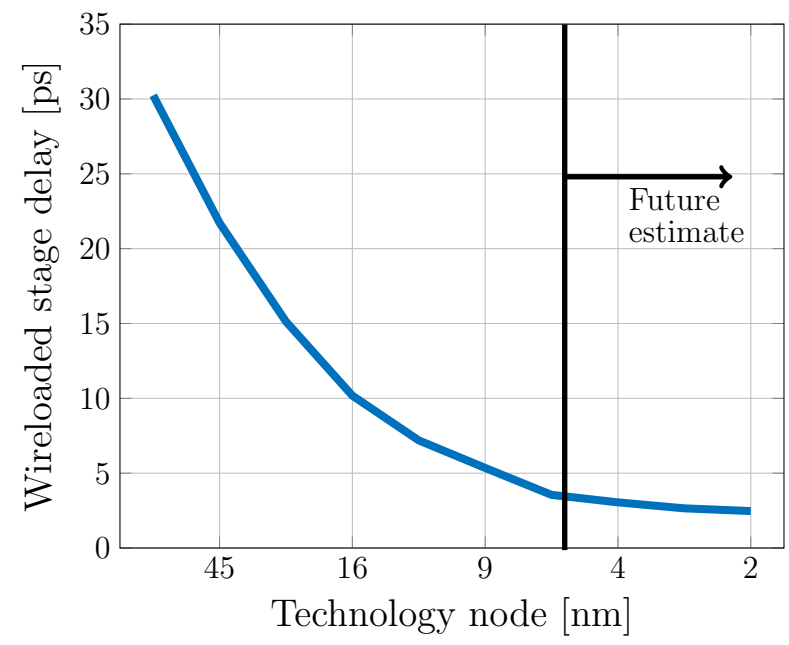

Fig. 9. Wire loaded stage delay of a logic device for different technology nodes [40]

In Figure 9, the evolution of the gate delay versus the technology size is shown. It can be seen that the decreasing trend of the gate delay starts to flatten towards the newer (smaller) nodes. This leads to believe that by only technology scaling, it will not be possible to decrease the conversion step size of TDCs further. Therefore, different techniques exist to reduce the conversion step size beyond the gate delay of an inverter. One of those techniques, which can be used in the locked loop architecture, is Local Passive Interpolation (LPI). In this technique, interpolation resistors are placed between successive delay elements to split the phase transition through the delay element, lowering the conversion step size even further [41]. However, due to the loading of the individual phase nodes, the interpolation is not linear when using equal resistors. This leads to an increased DNL error which is related to the drive strength of the delay cell. Individual tuning is often implemented.

To use a delay cell in the aforementioned loop structure, the delay element should be adjustable. This can be done in three ways: (1) by adjusting the supply current of the delay cell by placing an extra current limiting device in the supply branch of the delay element (Figure 10.a), (2) by modifying the supply voltage of the delay cell, (3) by adjusting the loading of the internal nodes by modifying the capacitance (Figure 10.b) [42]. In essence, all methods adjust the slew rate of the propagating signals, altering the propagation delay:

$$
T_{p d} \approx \frac{C_{L} \cdot V_{D D} / 2}{I_{D s a t}} .
$$

Where $I_{\text {Dsat }}$ represents the current of the devices in saturation, and $C_{L}$ represents the load capacitance which may or may not be parasitic.

To counteract the speed degradation of the delay elements due to ionising radiation, the delay elements should be able to

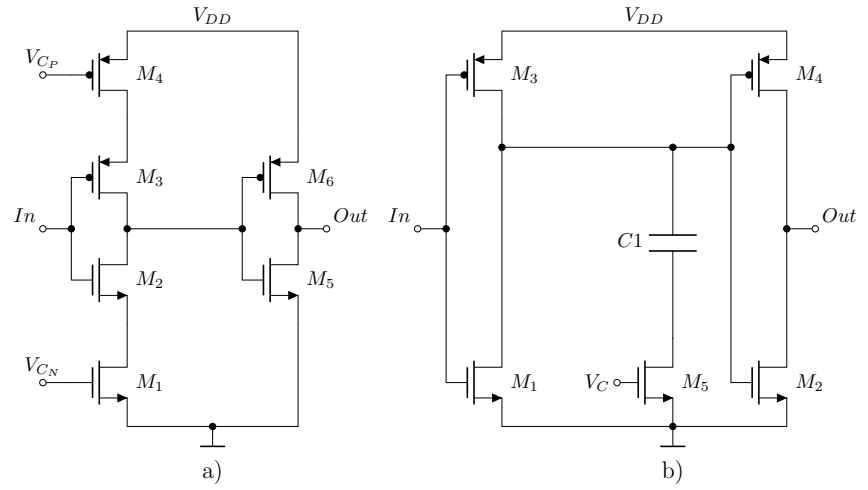

Fig. 10. (a) single ended current starved delay cell circuit. (b) single ended capacitive loaded delay cell circuit

reduce the propagation delay to counteract radiation damage. This means that a TDC using this type of locked loop architecture cannot achieve the smallest conversion step size of the technology node compared to architectures without compensation, as previously mentioned, since additional tuning margin is needed to counteract radiation degradation. Similar to the $\mathrm{CP}$, the compensation dynamic range may not be symmetrical because the permanent degradation of ionising radiation will increase the propagation delay.

The power consumption of these architectures mainly originates from the rapidly switching delay line. Therefore, designing a power-efficient delay cell is essential. Moreover, depending on the type of delay element, increasing the speed of the delay element to compensate for the degradation will also result in an increased switching current. Nevertheless, contributions from the feedback loop, the digital readout electronics and counters for possibly increasing the dynamic range may not be neglected ,especially for multi-channel architectures where the power consumption contribution from the clock distribution to all sample registers becomes significant.

The last important property of this architecture is the sampling frequency. For TDCs, the sample period is limited to the maximum measurable time. This means that when a TDC can measure a delay of $1 \mu \mathrm{s}$, the sampling frequency is limited to $1 \mathrm{MHz}$. However, a TDC architecture may be able to have a larger sampling frequency for small input delays. This is commonly the case for TDCs which trigger an interrupt when an input time event has been measured [43] or have multiple measurement channels.

\section{VERnier ARCHITECTURE}

The Vernier architecture can also achieve a conversion step size below the gate delay of the technology. This is accomplished by using the time difference of two delay cells with different propagation delays. As shown in Figure 11, the start signal propagates through a delay line of the slower delay elements (grey) and the stop signal through a fast delay line (white). The faster stop signal will trigger the sample registers at every node to save the state of the propagating start signal. Because the stop signal is faster than the start signal, it will catch up with the start signal, which can be seen as a state 


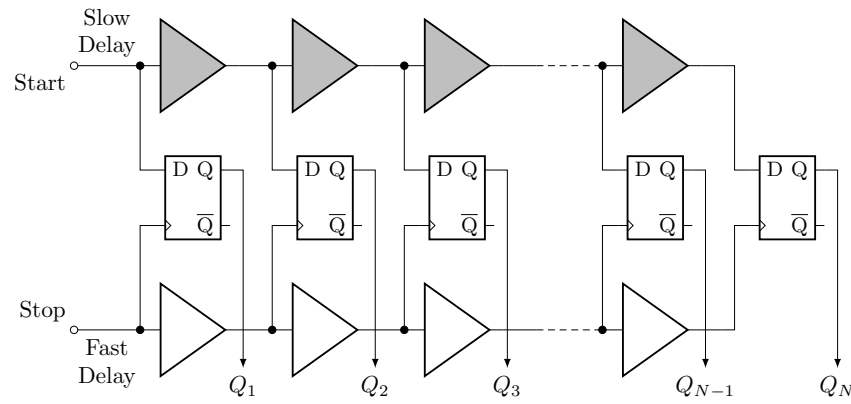

Fig. 11. Linear Vernier TDC architecture.

change in the output of the sample registers. The location of this state change represents the time difference between the start and stop signal.

Theoretically, it is possible to achieve an infinitely small conversion step size. However, due to device mismatch and random noise, the minimum resolution is still limited. A secondary effect when using this architecture is the larger sampling delay latency. In the previous architectures, the time it took to measure a timing event was equal to the timing event itself. This is not the case in this architecture, as both the start and stop signals are delayed. This sample delay has a significant impact on the conversion time of the converter. Using Equation 2, the conversion time can be calculated for a given resolution via:

$$
T_{\text {conv }}^{\text {Vernier }}=\frac{t_{d 1}}{T_{L S B}} \cdot T=\frac{t_{d 1}}{t_{d 1}-t_{d 2}} \cdot T .
$$

Where $t_{d 1}$ and $t_{d 2}$ correspond to the buffer delays and $T$ corresponds to the measured input delay.

By using replica delay lines, both delay lines can be compensated. This makes the architecture more robust for harsh environments. As the delay lines need to lock to a different bin size, the number of delay cells in each replica delay line can be different to introduce a bin size difference without the need for two different reference clocks.

Due to the rather small dynamic range for a relatively large number of delay cells and an even larger amount for an added compensation loop, this architecture is less suitable to be used as the primary time converter. However, as part of a hierarchical TDC architecture, it is perfectly suitable for fine time quantization.

An improved vernier TDC architecture is proposed in [44], where a larger dynamic range is accomplished for an equal amount of delay cells. In this two dimensional Vernier architecture, two delay lines, propagating the start and stop signal respectively, are placed orthogonally to each other to form the rows and columns of a two-dimensional plane. In this plane, the intersection points between the two delay lines represent different time delays similar to the linear Vernier TDC. However, the dynamic range can be significantly larger. The architecture shown in Figure 12, can achieve a dynamic range of $27 \Delta$ with an $8 \cdot 5 \Delta$ delay line, comparing this to a linear Vernier TDC, where it would only result in an $8 \Delta$ range. However, due to the two-dimensional plane, the layout becomes increasingly complex. It introduces a significant

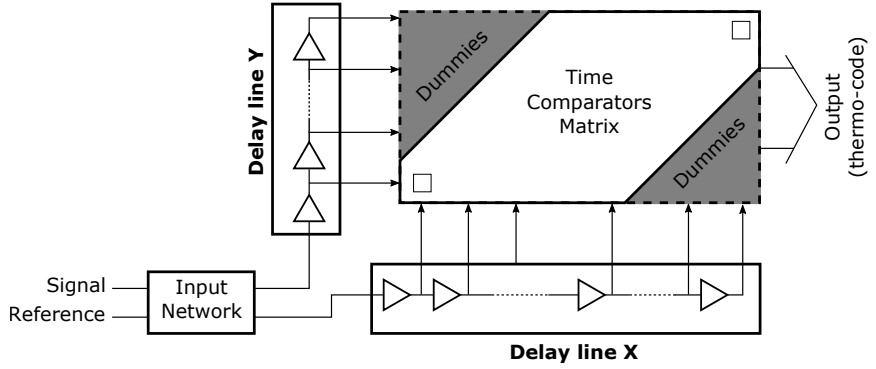

Fig. 12. Two dimensional Vernier TDC architecture [44].

amount of parasitic capacitive loading on the nodes of the delay elements as multiple sample comparators are connected to the same delay stage, which is not the case for the linear Vernier TDC.

Stabilising the mentioned architecture with a feedback loop to compensate degradation from harsh environment applications requires both delay lines to be compensated. This is done similarly to the linear Vernier TDC mentioned before. As this architecture is more efficient due to the increased measurement range, a compensation loop has become a more valid choice.

An even more improved Vernier architecture is presented in [45], where the delay lines are replaced by an oscillator which can be disabled. The benefit of using oscillators instead of delay lines is the ability to use multiple laps of the oscillators to increase the measurement range without the need for more delay stages and makes it possible to use the entire twodimensional plane, which is not the case in [44]. As there are fewer delay elements used, the power consumption will be lower. Moreover, by gating the ring oscillators, the average power consumption also reduces significantly.

\section{OVERSAMPLING TDC}

The last architectures are oversampling TDCs. In such architectures, multiple samples are used to measure the input time difference. This kind of architecture can not be used in single-shot applications but instead is used in applications with repetitive time-based signals with relatively low bandwidth. As these applications are also found in harsh environments, for instance, in radiation-hardened ADPLLs for frequency synthesis, time-based ADCs or TOF measurements, two architectures are presented which have proven to work in these circumstances.

\section{A. Multipath GRO TDC}

The multipath gated ring oscillator (GRO) TDC is an oversampling TDC where the ring oscillator is gated (stopped) between measurements, resulting in a more power-efficient architecture. As shown in Figure 13, the architecture mainly consists of the GRO and the Time Difference Generator (TDG). The TDG circuit converts the two separate start and stop inputs into a single enable signal used to gate the GRO. When a timing event arrives, the TDG will enable the GRO, which resumes oscillation. When the stop signal arrives, the TDG will disable the GRO causing the current phase of the oscillator to be frozen inside the GRO itself. In this sense, the GRO can remember its phase. At this time, a sample 


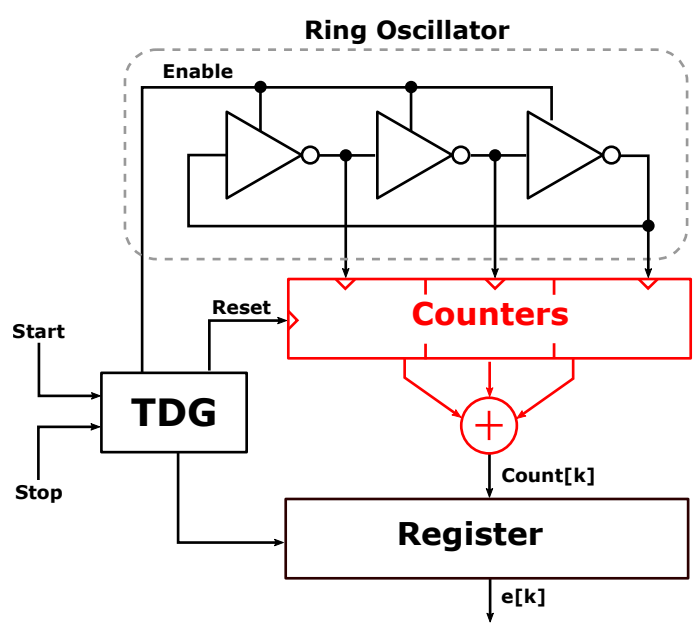

Fig. 13. Gated ring oscillator TDC architecture [46].

register samples the phase of the GRO and stores it. On the arrival of the next event, the TDG enables the GRO again, which causes the oscillator to start from its previously frozen phase, taking the quantization error from the last sample with it. When the stop signal arrives, the oscillator stops again, and another sample of the oscillator is made. Taking the difference of both samples will result in the measured time difference. As the second sample also contains the quantization error from the first sample, which is subtracted, the quantization noise of this architecture is first-order noise-shaped similar to a firstorder $\Sigma \Delta$ modulator. This noise shaping ability is helpful for repetitive measurements, such as LIDAR or phase-detection in ADPLLs. This TDC is less suitable for particle detection since only one shot is available to measure an event.

The multipath property of this architecture mentioned in [46], uses multiple phases from preceding stages to increase the transitioning speed of a single stage. This approach results in a faster ring oscillator and an improved resolution beyond the gate delay of the technology. However, this comes with a cost of increased power as described in [31].

In this architecture, it is not easy to include the GRO in a control loop. Due to the gated principle, the time the GRO is enabled is too short and unpredictable to lock a feedback loop. Therefore, periodically measuring the raw resolution of the converter and running a CDT to measure the non-linearity is still required to achieve correct output data.

As it is a common factor in all architectures mentioned above to sample the state of a delay line or multiple delay cells, a single event may disturb the measured state. An SEU can cause a bit to change its state resulting in multiple state transitions present in the delay line sample, resulting in an incorrect output result. A technique to mitigate the SEU is presented in [47]. With this technique, the so-called bubble problem is solved by filtering single glitches in the delay line sample using a triple input NAND gate to eliminate single bit bubbles separated at least three bits apart. Another possible solution mentioned in this article is to count the number of one's in the delay line sample. As this is possible in a linear Vernier type architecture, which outputs a thermometric code, it is not possible in circular delay line architectures like the

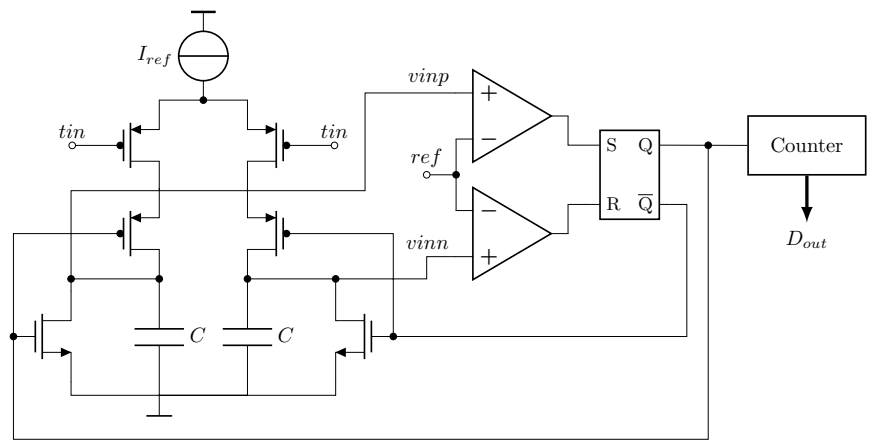

Fig. 14. first order delta-sigma TDC architecture [48].

DLL, PLL and GRO TDC, as the amount of ones and zeroes is always the same.

A single event can also cause the locked loop structure to unlock. For a PLL architecture, this has a significant impact as the error will accumulate inside the loop and requires the loop to resettle, which may take several microseconds. This accumulation is not the case for a DLL architecture as the reference clock cycle is refreshed every clock cycle, which causes the DLL to recover more quickly.

\section{B. MASH Delta Sigma TDC}

As the $\Delta \Sigma$ ADC architectures are well known to be stable architectures for environmental noise and component mismatch, this also translates to $\Delta \Sigma$ TDC architectures. Presented in [48] a third-order $\Delta \Sigma$ TDC is shown achieving a $10.5 \mathrm{ps}$ time resolution up to a TID of 3.4 MGy. The architecture is based on an error feedback loop, using a relaxation oscillator as a time integrator. Figure 14 shows a single stage of the architecture, representing a first-order $\Delta \Sigma$ TDC. Functionally, this architecture has a similar operation as the GRO TDC architecture. The oscillator phase is saved in between measurements causing the quantization error to be subtracted from the next sample, resulting in a first-order noise shaping. Several of these circuits are then cascaded where each subsequent stage quantizes the error of the previous one resulting in a higherorder noise shaping similar to a MASH $\Delta \Sigma$ ADC.

The stability of this architecture is mainly determined by the design of the oscillator. The oscillator is a relaxation type oscillator with a frequency that is mainly determined by passive components, making the oscillator more tolerant for PVT variation and ionising radiation. However, as the delay path through the comparators and the SR-latch also adds to the oscillator's period, degradation from ionising radiation can not be eliminated. Additionally, when multiple stages are placed in series to increase the order of quantisation noise shaping, a possible mismatch between the relaxation oscillators will introduce first- and second-order imperfections.

Another essential aspect determining this architecture's performance is the phase skew, which occurs when the oscillator phase is not perfectly preserved and causes the comparator to switch states at the wrong moment. As this skew also depends on the oscillator's phase itself, it behaves as added random noise that degrades the achievable effective resolution. As this 
effect is amplified due to ionising radiation, the ENOB of the converter will decrease with dose, which is also seen in [48]. A solution to this problem is proposed in [49] where a delay line is used to preserve the phase of the oscillator when the phase is held near the reference voltage of the comparator. As this solution proves to improve the noise, it was unfortunately not tested with TID effects.

\section{CONCLUSION}

This review article presented a couple of well-known TDC architectures evaluated for applications inside harsh environments. Each architecture has its unique characteristics. Therefore, no general compensation or calibration technique can be used for all TDC architectures.

In this article, different compensation strategies are presented for single-shot TDC architectures and oversampling TDC architectures. For a single-shot architecture, the accuracy of the individual measurements is more critical than oversampling architecture, where the random noise contributions are a more relevant design criterion.

It is also crucial to clearly understand the trade-off between the improved radiation tolerance and the reduced performance, such as power consumption, increased complexity, area and resolution. This trade-off is likely to be very applicationspecific. However, we found three main trade-offs that are very important for designing a radiation-tolerant TDC. The first trade-off lies between the conversion step size and the non-linearity error, as these are the main specifications that determine the TDC's performance. The second trade-off lies between the overall power consumption and the radiation tolerance. Choosing larger devices will reduce the effect of TID and SEE but may increase the overall power consumption. The last trade-off is a choice between a DLL or a PLL compensation architecture. The inherently stable DLL architecture can achieve lower random noise due to a fresh reference clock edge entering the delay line every cycle, therefore, not accumulating the random noise contribution inside the delay line, which is not the case for a ring oscillator in a PLL architecture. However, static phase offset due to charge pump current mismatch is more of a concern in a DLL architecture than a PLL architecture, for a PLL, only the phase relation between the reference clock and the VCO is affected, and not the frequency itself, which determines the TDC conversion step size.

Nevertheless, as there are not yet many publications about radiation assessments of TDCs, some of the presented articles show performance figures comparable with the current state of the art. The comparable specifications are the conversion step size which is mainly related to the used technology, and the non-linearity error determined by the sizes of the used devices. Other performance numbers strongly depend on the targeted application.

Additionally, many different compensation techniques for PVT variations like the charge pump compensation and locked loop architectures can be implemented to improve radiation tolerance, which means that PVT-robust TDC architectures are likely to also perform well in radiation environments. To conclude on which architecture performs best under ionising radiation, it is believed that the PLL type architecture offers the best combination of robustness and intrinsic performance. When using passive interpolations and an added counter, a large dynamic range and a stable conversion step size can be achieved, which are two properties that are important in almost all applications.

\section{REFERENCES}

[1] G. S. Stanford, "An introduction to multichannel analyzers," Nuclear Instruments and Methods, vol. 34, pp. 1-9, 1965.

[2] S. Tancock, E. Arabul, and N. Dahnoun, "A review of new time-todigital conversion techniques," IEEE Transactions on Instrumentation and Measurement, vol. 68, no. 10, pp. 3406-3417, 2019.

[3] A. J. Annema, B. Nauta, R. van Langevelde, and H. Tuinhout, "Analog circuits in ultra-deep-submicron cmos," IEEE Journal of Solid-State Circuits, vol. 40, no. 1, pp. 132-143, 2005.

[4] J. Jansson, P. Keränen, J. Kostamovaara, and A. Baschirotto, "Cmos technology scaling advantages in time domain signal processing," in 2017 IEEE International Instrumentation and Measurement Technology Conference (I2MTC), 2017, pp. 1-5.

[5] M. Baert and W. Dehaene, "A 5-gs/s 7.2-enob time-interleaved vcobased adc achieving $30.5 \mathrm{fj} / \mathrm{cs}$," IEEE Journal of Solid-State Circuits, vol. 55, no. 6, pp. 1577-1587, 2020.

[6] M. N. Ullah, E. Pratiwi, J. Cheon, H. Choi, and J. Y. Yeom, "Instrumentation for time-of-flight positron emission tomography," Nuclear Medicine and Molecular Imaging, vol. 50, no. 2, pp. 112-122, Jun 2016.

[7] J. M. Ollinger and J. A. Fessler, "Positron-emission tomography," IEEE Signal processing magazine, pp. 43-55, 1997.

[8] H. A. Shakhawat Hossen Prayash, M. Ragib Shaharear, M. F. Islam, S. Islam, N. Hossain, and S. Datta, "Designing and optimization of an autonomous vacuum floor cleaning robot," 2019 IEEE International Conference on Robotics, Automation, Artificial-Intelligence and Internetof-Things, RAAICON 2019, pp. 25-30, 2019.

[9] R. B. Staszewski, J. L. Wallberg, S. Rezeq, Chih-Ming Hung, O. E. Eliezer, S. K. Vemulapalli, C. Fernando, K. Maggio, R. Staszewski, N. Barton, Meng-Chang Lee, P. Cruise, M. Entezari, K. Muhammad, and D. Leipold, "All-digital pll and transmitter for mobile phones," IEEE Journal of Solid-State Circuits, vol. 40, no. 12, pp. 2469-2482, 2005.

[10] Z. Flowers, D. W. Kang, Q. Meier, S. C. Park, and C. Rogan, "Timing information at hl-lhc: complete determination of masses of dark matter and long lived particle," Journal of High Energy Physics, vol. 2020, no. 3, p. 132, Mar 2020.

[11] O. Cerri, S. Xie, C. Pena, and M. Spiropulu, "Identification of longlived charged particles using time-of-flight systems at the upgraded lhc detectors," Journal of High Energy Physics, vol. 2019, no. 4, p. 37, Apr 2019.

[12] F. Sauli, "The gas electron multiplier (GEM): Operating principles and applications," Nucl. Instrum. Meth. A, vol. 805, pp. 2-24, 2016.

[13] F. B. McLean and T. R. Oldham, "Basic mechanisms of radiation effects in electronic materials and devices," HARRY DIAMOND LABS ADELPHI MD, Tech. Rep., 1987.

[14] F. Faccio and G. Cervelli, "Radiation-induced edge effects in deep submicron cmos transistors," IEEE Transactions on Nuclear Science, vol. 52, no. 6, pp. 2413-2420, 2005.

[15] F. Faccio, S. Michelis, D. Cornale, A. Paccagnella, and S. Gerardin, "Radiation-induced short channel (risce) and narrow channel (rince) effects in 65 and $130 \mathrm{~nm}$ mosfets," IEEE Transactions on Nuclear Science, vol. 62, no. 6, pp. 2933-2940, 2015.

[16] C. Zhang, F. Jazaeri, A. Pezzotta, C. Bruschini, G. Borghello, F. Faccio, S. Mattiazzo, A. Baschirotto, and C. Enz, "Characterization of gigarad total ionizing dose and annealing effects on 28-nm bulk mosfets," IEEE Transactions on Nuclear Science, vol. 64, no. 10, pp. 2639-2647, 2017.

[17] M. L. Alles, R. D. Schrimpf, R. A. Reed, L. W. Massengill, R. A Weller, M. H. Mendenhall, D. R. Ball, K. M. Warren, T. D. Loveless, J. S. Kauppila, and B. D. Sierawski, "Radiation hardness of fdsoi and finfet technologies," in IEEE 2011 International SOI Conference, 2011, pp. 1-2.

[18] J. Verbeeck, P. Leroux, and M. Steyaert, "Radiation effects upon the mismatch of identically laid out transistor pairs," in 2011 IEEE ICMTS International Conference on Microelectronic Test Structures, 2011, pp. 194-197. 
[19] P. E. Dodd, "Device simulation of charge collection and single-event upset," IEEE Transactions on Nuclear Science, vol. 43, no. 2, pp. 561$575,1996$.

[20] A. Al Youssef, L. Artola, S. Ducret, and G. Hubert, "Compact modeling of single-event latchup of integrated cmos circuit," IEEE Transactions on Nuclear Science, vol. 66, no. 7, pp. 1510-1515, 2019.

[21] P. Maillard, W. T. Holman, T. D. Loveless, B. L. Bhuva, and L. W. Massengill, "An rhbd technique to mitigate missing pulses in delay locked loops," IEEE Transactions on Nuclear Science, vol. 57, no. 6, pp. 3634-3639, 2010.

[22] R. C. Lacoe, "Improving integrated circuit performance through the application of hardness-by-design methodology," IEEE Transactions on Nuclear Science, vol. 55, no. 4, pp. 1903-1925, 2008.

[23] W. Snoeys, F. Faccio, M. Burns, M. Campbell, E. Cantatore, N. Carrer, L. Casagrande, A. Cavagnoli, C. Dachs, S. Di Liberto, F. Formenti, A. Giraldo, E. H. M. Heijne, P. Jarron, M. Letheren, A. Marchioro, P. Martinengo, F. Meddi, B. Mikulec, M. Morando, M. Morel, E. Noah, A. Paccagnella, I. Ropotar, S. Saladino, W. Sansen, F. Santopietro, F. Scarlassara, G. F. Segato, P. M. Signe, F. Soramel, L. Vannucci, and $\mathrm{K}$. Vleugels, "Layout techniques to enhance the radiation tolerance of standard cmos technologies demonstrated on a pixel detector readout chip," Nuclear Instruments and Methods in Physics Research Section A: Accelerators, Spectrometers, Detectors and Associated Equipment, vol. 439, no. 2, pp. 349-360, 2000.

[24] W. J. Snoeys, T. A. P. Gutierrez, and G. Anelli, "A new nmos layout structure for radiation tolerance," in 2001 IEEE Nuclear Science Symposium Conference Record (Cat. No.01CH37310), vol. 2, 2001, pp. $822-826$ vol. 2 .

[25] V. Petrović, G. Schoof, and Z. Stamenković, "Fault-tolerant tmr and dmr circuits with latchup protection switches," Microelectronics Reliability, vol. 54, no. 8, pp. 1613-1626, 2014.

[26] Y. Aguiar, F. Wrobel, J.-L. Autran, P. Leroux, F. Saigné, V. Pouget, and A. Touboul, "Design exploration of majority voter architectures based on the signal probability for tmr strategy optimization in space applications," Microelectronics Reliability, vol. 114, p. 113877, 2020.

[27] J. Chen, S. Chen, Y. He, J. Qin, B. Liang, B. Liu, and P. Huang, "Novel layout technique for single-event transient mitigation using dummy transistor," IEEE Transactions on Device and Materials Reliability, vol. 13, no. 1, pp. 177-184, 2013.

[28] J. R. Ahlbin, L. W. Massengill, B. L. Bhuva, B. Narasimham, M. J. Gadlage, and P. H. Eaton, "Single-event transient pulse quenching in advanced cmos logic circuits," IEEE Transactions on Nuclear Science, vol. 56, no. 6, pp. 3050-3056, 2009.

[29] B. K. Swann, B. J. Blalock, L. G. Clonts, D. M. Binkley, J. M. Rochelle, E. Breeding, and K. M. Baldwin, "A 100-ps time-resolution cmos time-to-digital converter for positron emission tomography imaging applications," IEEE Journal of Solid-State Circuits, vol. 39, no. 11, pp. 1839-1852, Nov 2004.

[30] B. Van Bockel, J. Prinzie, and P. Leroux, "Design of a 4 ps radiation hardened tdc with an improved interpolation technique," Proceeding of science, vol. TWEPP2019, p. 127, 2020.

[31] B. Van Bockel, J. Prinzie, and P. Leroux, "Radiation assessment of a 15.6ps single-shot time-to-digital converter in terms of tid," Electronics, vol. 8, no. 5, p. 558, May 2019.

[32] J. Prinzie, J. Christiansen, P. Moreira, M. Steyaert, and P. Leroux, "Comparison of a $65 \mathrm{~nm}$ cmos ring- and lc-oscillator based pll in terms of tid and seu sensitivity," IEEE Transactions on Nuclear Science, vol. 64, no. 1, pp. 245-252, 2017.

[33] J. Prinzie, M. Steyaert, P. Leroux, J. Christiansen, and P. Moreira, "A single-event upset robust, $2.2 \mathrm{ghz}$ to $3.2 \mathrm{ghz}, 345 \mathrm{fs}$ jitter pll with triplemodular redundant phase detector in $65 \mathrm{~nm}$ cmos," 2016 IEEE Asian Solid-State Circuits Conference, A-SSCC 2016 - Proceedings, pp. 285288, 2017

[34] J. Prinzie, S. Kulis, P. Leitao, R. Francisco, V. De Smedt, P. Moreira, and P. Leroux, "A low noise fault tolerant radiation hardened 2.56 gbps clock-data recovery circuit with high speed feed forward correction in $65 \mathrm{~nm}$ cmos," in 2019 IEEE 10th Latin American Symposium on Circuits Systems (LASCAS), 2019, pp. 63-66.

[35] M. Menouni, M. Barbero, F. Bompard, S. Bonacini, D. Fougeron, R. Gaglione, A. Rozanov, P. Valerio, and A. Wang, "1-grad total dose evaluation of $65 \mathrm{~nm}$ cmos technology for the hl-lhc upgrades," Journal of Instrumentation, vol. 10, no. 5, 2015.

[36] Jae-Shin Lee, Min-Sun Keel, Shin-Il Lim, and Suki Kim, "Charge pump with perfect current matching characteristics in phase-locked loops," Electronics Letters, vol. 36, no. 23, pp. 1907-1908, 2000.

[37] V. Kalenteridis, K. Papathanasiou, and S. Siskos, "Analysis and design of charge pumps for telecommunication applications," in VLSI-SoC: Design
Methodologies for SoC and SiP, C. Piguet, R. Reis, and D. Soudris, Eds. Springer Berlin Heidelberg, 2010, pp. 43-60.

[38] M. Moazedi, A. Abrishamifar, and A. M. Sodagar, "A highly-linear modified pseudo-differential current starved delay element with wide tuning range," 2011 19th Iranian Conference on Electrical Engineering, ICEE 2011, 2011.

[39] J. G. Maneatis and M. A. Horowitz, "Precise delay generation using coupled oscillators," Digest of Technical Papers - IEEE International Solid-State Circuits Conference, vol. 28, no. 12, pp. 118-119, 1993.

[40] "More Moore," IRDS, Tech. Rep., 2016.

[41] S. Henzler, S. Koeppe, D. Lorenz, W. Kamp, R. Kuenemund, and D. Schmitt-Landsiedel, "A local passive time interpolation concept for variation-tolerant high-resolution time-to-digital conversion," IEEE Journal of Solid-State Circuits, vol. 43, no. 7, pp. 1666-1676, 2008

[42] J. Prinzie, M. Steyaert, and P. Leroux, "A single shot tdc with 4.8 ps resolution in $40 \mathrm{~nm}$ cmos for high energy physics applications," Journal of Instrumentation, vol. 10, no. 1, pp. 0-8, 2015.

[43] TDC7200 Time-to-Digital Converter for Time-of-Flight Applications in LIDAR, Magnetostrictive and Flow Meters, Texas Instruments, 2016, rev. B. [Online]. Available: https://www.ti.com/product/TDC7200

[44] A. Liscidini, L. Vercesi, and R. Castello, "Time to digital converter based on a 2-dimensions vernier architecture," in 2009 IEEE Custom Integrated Circuits Conference, 2009, pp. 45-48.

[45] P. Lu, P. Andreani, and A. Liscidini, "A 2-d gro vernier time-to-digital converter with large input range and small latency," Digest of Papers - IEEE Radio Frequency Integrated Circuits Symposium, pp. 151-154, 2013.

[46] M. Z. Straayer and M. H. Perrott, "A multi-path gated ring oscillator tdc with first-order noise shaping," IEEE Journal of Solid-State Circuits, vol. 44, no. 4, pp. 1089-1098, 2009.

[47] E. Sall, M. Vesterbacka, and K. O. Andersson, "A study of digital decoders in flash analog-to-digital converters," in 2004 IEEE International Symposium on Circuits and Systems (IEEE Cat. No.04CH37512), vol. 1, 2004, pp. I-I.

[48] Y. Cao, W. De Cock, M. Steyaert, and P. Leroux, "Design and assessment of a 6 ps-resolution time-to-digital converter with 5 MGy gamma-dose tolerance for lidar application," IEEE Transactions on Nuclear Science, vol. 59, no. 4 PART 2, pp. 1382-1389, 2012.

[49] Y. Cao, W. De Cock, M. Steyaert, and P. Leroux, "1-1-1 mash $\delta \sigma$ timeto-digital converters with 6 ps resolution and third-order noise-shaping," IEEE Journal of Solid-State Circuits, vol. 47, no. 9, pp. 2093-2106, 2012. 